\title{
Associations between socioemotional alterations, quality of life, and social functioning in multiple sclerosis: A scoping review
}

\author{
Marialaura Di Tella ${ }^{1} \cdot$ Marinella Clerico $^{2} \cdot$ Lorys Castelli $^{1}$
}

Accepted: 7 October 2021

(c) The Author(s) 2021

\begin{abstract}
The main aim of the present scoping review is to systematically review the available studies that investigated the associations between socioemotional alterations (i.e., social cognition impairments/alexithymia/difficulties in emotion regulation) and both reduced QoL and social functioning in patients with Multiple Sclerosis (MS). The articles were selected from the PubMed, PsycINFO, and Scopus databases. The main exclusion criteria were qualitative studies, articles that did not use validated instruments, and studies that did not investigate the association between socioemotional skills and QoL/social functioning in MS. Of the eight studies fulfilling the inclusion criteria, six found significant associations between social cognitive and emotion regulation abilities and QoL/social functioning in patients with MS, while two found no significant relationships particularly between the performance on social cognition tasks and QoL measures. Overall, the majority of findings seem to highlight that socioemotional alterations contribute to impaired QoL and social functioning in MS. However, given the still limited evidence, future studies are needed to replicate and confirm the available results, paying attention to two principal aspects: the use of standardized and ecological tasks for the assessment of social cognition skills and the recruitment of samples involving patients with different types of MS.
\end{abstract}

Keywords Multiple sclerosis $\cdot$ Social cognition $\cdot$ Emotion regulation $\cdot$ Alexithymia $\cdot$ Quality of life $\cdot$ Social functioning

\section{Introduction}

Multiple sclerosis (MS) is a chronic inflammatory and neurodegenerative disease of the central nervous system, which affects approximately 2.5 million individuals all over the world (Files et al., 2015). It is usually diagnosed between the age of 20 and 40 and its prognosis is often unpredictable. Depending on the location and extent of lesions, patients may experience different disease-related stressful conditions, such as motor weakness, sensory deficit, impaired balance, and urinary disturbance.

In addition to physical symptoms, patients with MS often report cognitive impairments (Calabrese, 2006) and high levels of psychological distress (Feinstein, 2004; Siegert \&

Marialaura Di Tella

marialaura.ditella@unito.it

1 Department of Psychology, University of Turin, Via Verdi 10, 10124 Turin, Italy

2 Department of Clinical and Biological Sciences, School of Medicine, University of Turin, Azienda Ospedaliera Universitaria San Luigi Gonzaga, Turin, Italy
Abernethy, 2005). Particularly, psychiatric comorbidities can occur in up to $95 \%$ of patients with MS. Depression is the most common condition with a prevalence of around 50\%, followed by anxiety, which ranges from 14 to $41 \%$ (Paparrigopoulos et al., 2010).

Similarly, cognitive decline has been reported in up to $65 \%$ of patients with MS (Benedict et al., 2017; Messinis et al., 2018). Main signs of cognitive impairment usually include deficits in information processing speed, verbal and visuospatial memory, verbal fluency, and executive functions (Planche et al., 2015; Prakash et al., 2008).

More recently, researchers have started to focus their attention also on the investigation of social and interpersonal aspects of cognition (i.e., social cognition) in patients with MS. Social cognition has been defined as "the ability to construct representations of the relation between oneself and others and to use those representations flexibly to guide social behavior' (p. 231, Adolphs, 2001). Examples of social cognition abilities include both the capacity to represent other people's intentions and beliefs (i.e., Theory of Mind, ToM; Premack \& Woodruff, 1978; 
Leslie, 1987), and the ability to share and recognize the emotions of others (Lieberman, 2007).

Deficits in social cognition have been reported in a number of clinical conditions, such as autism spectrum disorder, schizophrenia, neurodegenerative disorders and chronic pain conditions (e.g., Senju, 2013; Bora et al., 2015; Di Tella et al., 2015; Green et al., 2015), compromising the patient's ability to implement appropriate social interactions. Alterations in social cognition skills have also been linked to difficulties in both the ability to identify and describe one's own feelings (i.e., 'alexithymia'; Taylor et al., 1997), and the ability to correctly regulate one's own emotions (e.g., Le Berre, 2019; Di Tella et al., 2020).

All those competencies together are fundamental to obtain adequate social support from other people and to cope with chronic disabling conditions such as MS (Chalah \& Ayache, 2017a). Conversely, if these skills are compromised, people may find themselves struggling to interact effectively in interpersonal contexts, with negative consequences on Quality of Life (QoL) and social functioning (Krause et al., 2013; Schwartz \& Frohner, 2005). While QoL has been defined by the World Health Organization as an 'individuals' perception of their position in life in the context of the culture and value systems in which they live, and in relation to their goals, expectations, standards and concerns', social functioning usually refers to the ability to fulfill the individual's role within his/her different environments, such as work and social environments (e.g., Bosc, 2000).

Furthermore, social cognition deficits and alexithymia have been shown to be significantly associated with anxiety/ depressive symptoms (Luminet, 2009; Richards et al., 2002; Washburn et al., 2016), which in turn can contribute to the high prevalence of affective disorders observed in patients with MS.

Previous systematic reviews have been carried out to address the magnitude and extension of social cognition impairments and alexithymia in MS studies. The majority of those reviews have focused on the measurement and neuroanatomical correlates of social cognition and alexithymia (e.g., Bora et al., 2016; Cotter et al., 2016; Chalah \& Ayache, 2017b; Labbe et al., 2018), highlighting the presence of alterations in ToM (especially when assessed trough visual tasks), recognition of negative facial emotional expressions (anger and fear), and ability to adequately identify and describe one's own feelings.

However, the possible effects of these cognitive and emotional deficits on QoL and social functioning of patients with MS have not previously been integrated.

Therefore, the main aim of this scoping review is to systematically review the available studies that investigated the association between socioemotional alterations (i.e., social cognition impairments/alexithymia/difficulties in emotion regulation) and both reduced QoL and social functioning in patients with MS.

\section{Methods}

The aim of a scoping review is to identify and summarize key concepts in a particular research area. In contrast to a systematic review, the quality of evidence is not evaluated within a scoping review. Instead, broader topics and research questions are addressed with the purpose of identifying research gaps and making recommendations for future research. The present scoping review was conducted following the Preferred Reporting Items for Systematic reviews and Meta-Analyses extension for Scoping Reviews (PRISMAScR) checklist (Tricco et al., 2018). The protocol was not pre-registered.

\section{Search Strategy}

A comprehensive, systematic literature search was conducted between November 2020 and January 2021 in PubMed, PsycINFO, and Scopus databases. The following keywords combinations were used to identify target published articles: (social cognition OR emotion recognition OR theory of mind OR empathy OR alexithymia OR emotion regulation) AND (quality of life OR social functioning) AND multiple sclerosis.

The last run was conducted on the $14^{\text {th }}$ January 2021. With the use of this search string, 1.270 titles were initially identified from 1942 up until 2021 (see Fig. 1 for the flow diagram of article selection).

\section{Eligibility Criteria}

The present review aimed to identify peer-reviewed academic articles that investigated the relationship between social cognition/alexithymia/emotion regulation and QoL/ social functioning in patients with MS. Only academic peerreviewed scientific papers that were published in English were eligible for inclusion in this review.

Exclusion criteria were as follows:

1) Articles that exclusively assessed social cognition (i.e., ToM, empathy, and emotion recognition) impairments or alexithymia or emotion regulation, without providing further insights on the association between those deficits and QoL/social functioning in patients with MS.

2) Studies that used ad hoc constructed instruments or qualitative methods.

3) Articles that recruited and assessed patients with MS under 18 years of age or patients with MS together with patients with other neurological conditions. 
Fig. 1 Preferred Reporting Items for Systematic Reviews and Meta-Analyses (PRISMA) flow diagram for the study selection

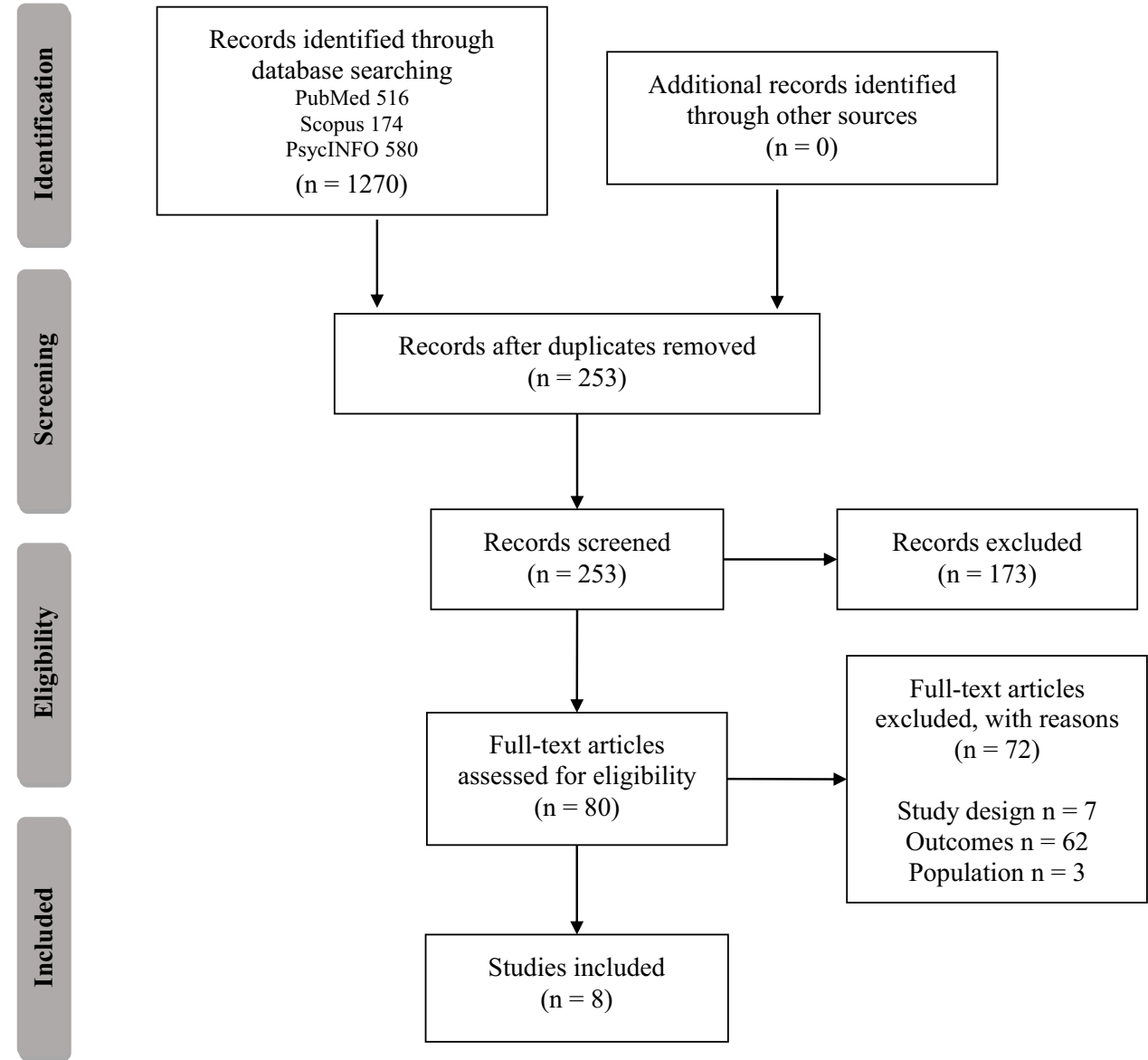

4) Articles published but not peer reviewed or under review at the time the search was carried out.

5) Textbooks and book chapters were also excluded, because many textbooks and book chapters do not provide new insights, as original research papers do, and not all of them are peer reviewed.

\section{Study Selection}

Study selection was carried out by two authors (MDT and LC), who first screened the titles and abstracts of all the retrieved articles and then examined the full text of all remaining publications. Disagreements on the inclusion or exclusion of publications were discussed until agreement was reached. Reference lists of relevant articles were also checked, in order to obtain additional important publications that were not included yet.

\section{Data Extraction}

The characteristics of all included studies were extracted by one author (MDT). Data items that were extracted from each included study were: author and year of publication, participants, methods, and main findings.

\section{Results}

\section{Study Selection}

After duplicates were removed, a total of 253 records were screened. Of these articles, 245 titles have been excluded based on title, abstract, and full text screening (Fig. 1). Most of these excluded articles did not assess social cognition skills (ToM, emotion recognition, and empathy), alexithymia or difficulties with emotion regulation in patients with MS $(n=173)$ or did not evaluate those constructs with regard to QoL/social functioning $(n=62)$ or did not use a research design that allowed to investigate those associations $(n=7)$. The remaining articles $(n=3)$ enrolled mixed samples of patients, including people with MS and other neurological conditions. The study selection process resulted in 8 studies that fulfilled the inclusion criteria and were included in the present review.

\section{Characteristics of Included Studies}

A summary of the main characteristics and results of the 8 studies included in the present review is provided in Table 1. The selected articles were published from 2009 to 2020 , 


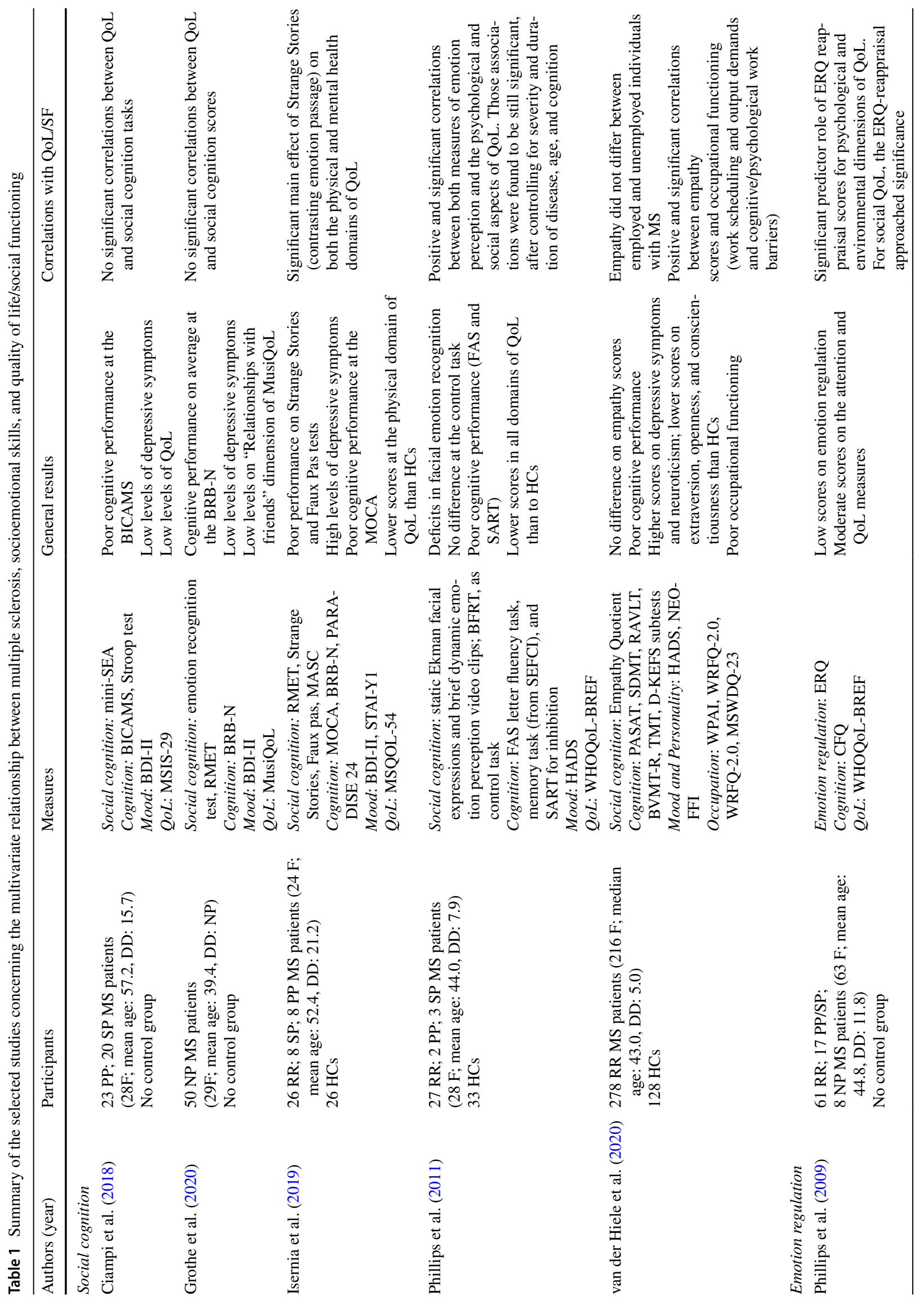




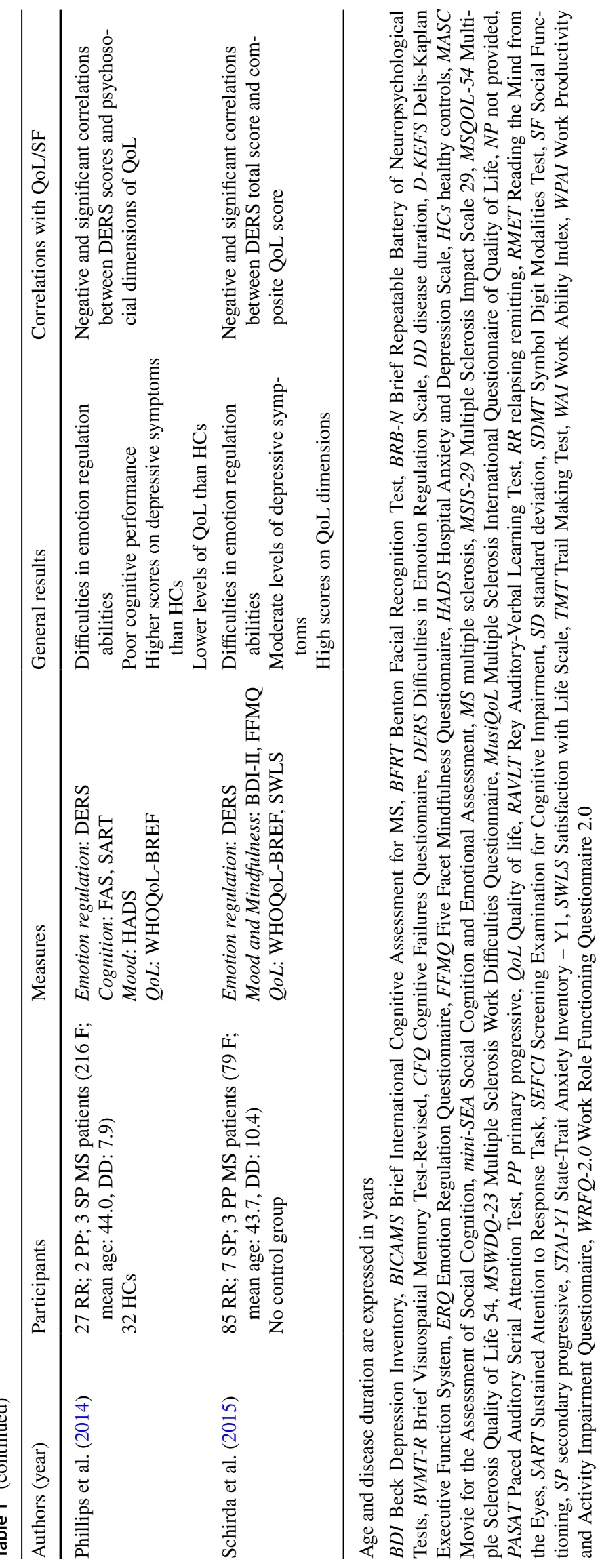


with half of the studies being carried out in the last four years $(n=4)$.

Most of articles reported about the type of MS and disease severity. Research samples typically include a combination of people with relapsing and progressive types of MS and involve patients with various levels of disability.

Based on the different aspects the selected articles focused on, we differentiated between studies that investigated the relationship between social cognition skills (i.e., ToM, emotion recognition, and empathy) and QoL/social functioning ( $n=5)$, and studies that examined the association between alexithymia/emotion regulation and QoL/social functioning $(n=3)$. The two article categories are presented below, separately.

\section{Social Cognition, Quality of Life, and Social Functioning in Multiple Sclerosis}

Among the studies that investigated the presence of social cognition deficits in patients with MS, five articles included in their assessment also the analysis of QoL and social functioning aspects. Those studies obtained, at least in part, distinct results. Indeed, two studies did not find significant correlations between lowered social cognition and QoL scores (Ciampi et al., 2018; Grothe et al., 2020), while the remaining studies found significant associations between reduced social cognition performance and worse QoL (Isernia et al., 2019; Phillips et al., 2011) or between altered social cognition and reduced social functioning (van der Hiele et al., 2020).

Particularly, the study of Ciampi et al. (2018) employed the mini-Social Cognition and Emotional Assessment (miniSEA) (Bertoux et al., 2012, 2014) to assess different aspects of social cognition in a group of patients with progressive types of MS $(n=43)$. The mini-SEA consists of two different tests, namely a shortened version of the Faux-Pas test and the Face Emotion Recognition test. The Faux Pas test includes both short passages, in which a character inadvertently hurts or offends another, and control stories, in which no faux pas is committed. The individual is required to identify if the faux pas has been made, why the main character did it (cognitive ToM) and how the victim of the faux pas must have felt (affective ToM). The Face Emotion Recognition test consists of 35 pictures, representing the six basic emotions (i.e., happiness, sadness, anger, surprise, fear, and disgust) and neutral expressions. The individual is asked to select the correct option for each of the presented photographs.

Among the different objectives the study pursued, the association between social cognition skills and QoL is of particular interest to the present review. QoL aspects have been assessed by means of the Multiple Sclerosis Impact Scale (MSIS-29; Hobart et al., 2001), which is a 29-item self-report questionnaire consisting of 20 items associated with the physical dimension of QoL and 9 items associated with the psychological component of QoL. Results of correlation analyses showed no significant association either between mini-SEA scores and QoL scores, or between faux pas and face emotion recognition subtest scores and MSIS29 scores.

Similarly, the study of Grothe et al. (2020) investigated the associations between social cognition abilities, clinical variables, and QoL in a cohort of patients with MS $(n=50)$. For the assessment of social cognition skills, the authors administered the following two tests: (1) a facial morphing task to assess emotion recognition, which consists of 48 morphing sequences (from neutral to the target expression), presented in random order (Lischke et al., 2012). The participants have been required to press a button when they had recognized one of the possible four emotions (i.e., happy, angry, fearful, sad); (2) the Reading the Mind in the Eyes (RMET; Baron-Cohen et al., 2001) to evaluate affective ToM, which requires the individual to choose the correct mental state among four alternatives that are provided below each of the 36 images the test is made of. With regard to QoL evaluation, the Multiple Sclerosis International Questionnaire of Quality of Life (MusiQoL; Simeoni et al., 2008) was administered. It allows the investigation of nine dimensions of QoL, which are specifically related to MS.

In order to examine the relationships between social cognition measures and QoL dimensions, correlation analyses were performed. Results showed the presence of non-significant associations between social cognition tasks and MusiQoL total or subscale scores.

Different results were found by the studies of Isernia et al. (2019) and Phillips et al. (2011). Particularly, Isernia et al. (2019) aimed to investigate the relationship between ToM, clinical variables, and neuropsychological deficits in a sample of patients with both relapsing and progressive types of MS $(n=42)$, compared to a group of healthy controls (HCs; $n=26)$. For the assessment of cognitive and affective ToM processes, the authors employed the following 4 tasks: (1) the RMET (Baron-Cohen et al., 2001); (2) the Strange Stories test (Happé, 1994), which includes both mental and physical passages that require the participant to infer characters' mental states or to make global inferences that go beyond what was explicitly mentioned in the text, respectively. For mental stories both total score and single-story scores have been considered; (3) Faux pas test (Baron-Cohen et al., 1999), of which the authors selected 4 faux pas and 4 matched control stories; (4) the Movie for the Assessment of Social Cognition (MASC) (Dziobek et al., 2006), which consists of 15-min video clips representing an ecological situation in which four friends meet to have dinner together. Video is interrupted several times to ask participants multiple-choice questions on emotions, thoughts, and intentions 
of the different characters involved in the story. In addition to ToM tasks, the Multiple Sclerosis Quality of Life 54 questionnaire was administered for the assessment of QoL dimensions (Vickrey et al., 1995).

With particular regard to social cognition and QoL, the authors tested whether a poor ToM performance was related to low levels of QoL. General Linear Model was performed with QoL scores as within-subject factor and ToM scores as between-subject factor. Results of GLM showed a main effect of contrasting emotion passage of the Strange Stories on both the physical and mental components of QoL. No other significant association between ToM tasks and QoL measure was detected. Those findings suggest a significant role that affective ToM seems to play in different areas of QoL.

Yet the study of Phillips et al. (2011) examined the ability to identify emotional and non-emotional information and whether difficulties in emotion perception were related to QoL in a cohort of patients with both relapsing and progressive types of MS $(n=32)$ compared to a group of HCs $(n=33)$. To assess emotion perception, the authors administered the following four tests: (1) static images of facial emotion, taken from the standard set of Ekman facial expressions, were used to assess the ability to correctly identify expressions of the six basic emotions (Young et al., 2002); (2) the Benton facial identity recognition test (Benton et al., 1983) was included, as a control task, to examine non-emotional aspects of facial perception; (3) dynamic emotion perception video task (Slessor et al., 2007), in which participants were asked to select the word that best describes the feelings of the main character in 16 videos; (4) dynamic age and gender perception video task was employed as a control task for the dynamic emotion perception task. With regard to QoL, the authors administered the World Health Organization quality of life questionnaire (WHOQoL-BREF; Skevington et al., 2004) to investigate both scores of patients with MS on four domains of QoL (i.e., Physical, Psychological, Social, and Environmental) and their relationship with difficulties in emotion perception. Results showed that people with MS rated their QoL as poorer than HCs in all domains of functioning. Furthermore, correlation analyses revealed that both measures of emotion perception were strongly related to psychological and social domains of QoL, but not to the physical domain. Perceiving emotions from videos was also associated with the environmental domain of the WHOQoL-BREF. Correlations between emotion perception and QoL were found to be still significant after controlling for disease severity and duration, age, and cognition, suggesting a specific pattern of associations between difficulties in emotion perception and poor social and psychological QoL.

Finally, the study of van der Hiele et al. (2020) investigated the possible associations between empathy and cognitive, psychological, and occupational functioning in a group of patients with relapsing-remitting MS $(n=278)$ compared to HCs $(n=128)$. For the assessment of empathy the authors administered to all participants the Empathy Quotient (Baron-Cohen \& Wheelwright, 2004), which is a 60 item self-report questionnaire with total score ranging from 0 to 80 (higher scores indicate greater empathic abilities), while for the evaluation of occupational functioning various measures have been employed to investigate different work-related aspects (e.g., self-reported influence of MS symptoms on productivity while at work; work role functioning; current work ability as compared to lifetime best; psychological/cognitive, physical, and external work difficulties). With particular regard to the association between empathy and occupational functioning, no significant differences were detected between employed and unemployed individuals with MS on empathy scores. However, results of correlation analyses showed the presence of significant associations between higher empathy scores and better occupational functioning, in terms of work scheduling and output demands, and less cognitive/psychological work barriers in patients with MS. These findings suggest that reduced empathy could be negatively associated with worse occupational functioning, with negative consequences on the individual's ability to perform complex activities of daily life.

\section{Alexithymia, Emotion Regulation, Social Functioning, and Quality of Life in Multiple Sclerosis}

Similar to social cognition skills, only three articles included in their assessment the analysis of QoL dimensions in relation to emotion regulation abilities (Phillips et al., 2009, 2014; Schirda et al., 2015). Conversely, to the best of our knowledge, no study has investigated the relationship between alexithymia and either social functioning or QoL in patients with MS yet. Likewise, we did not retrieve any article that examined the association between emotion regulation abilities and social functioning.

The three available articles yielded similar results in terms of significant associations that were detected between difficulties in emotion regulation and reduced QoL. Particularly, the study of Phillips et al. (2009) mainly aimed to investigate the association between emotion regulation strategies and QoL in a group of people with both relapsing and progressive types of MS $(n=86)$. For the assessment of emotion regulation, the authors employed the Emotion Regulation Questionnaire (ERQ; Gross \& John, 2003), which allows to evaluate two different types of emotion regulation strategies, that is reappraisal and expressive suppression, while for the assessment of QoL they administered the WHOQoL-BREF (Skevington et al., 2004). In order to examine the relationships between QoL dimensions and emotion regulation abilities, a series of regression analyses were performed. Results 
showed that ERQ-reappraisal significantly predicted poor psychological QoL and environmental QoL, while for social QoL, ERQ-reappraisal approached significance $(p=0.06)$. Conversely, expressive suppression was not found to be a significant predictor of any of QoL dimensions. These findings seem to highlight that failure to use adequate emotion regulation strategies may contribute to reduced QoL in patients with MS.

Similar findings were obtained by the study of Phillips et al. (2014), which analyzed the associations between emotion regulation difficulties and other factors including QoL in a group of patients with both relapsing and progressive types of MS $(n=31)$ compared to HCs $(n=31)$. Emotion regulation difficulties were assessed by means of the Difficulties in Emotion Regulation Scale (DERS; Gratz \& Roemer, 2004), which is a self-report questionnaire designed to assess multiple aspects of emotion regulation, while the WHOQoL-BREF (Skevington et al., 2004) was employed for the assessment of QoL-related dimensions.

Results of comparisons between patients with MS and HCs revealed that the former reported greater difficulties in emotion regulation and poorer QoL compared to HCs. With regard to the association between emotion regulation and QoL, the authors found that higher scores at the DERS total were significantly correlated with reduced social, psychological, and environmental dimensions of QoL in the MS group only, suggesting that these patients may experience greater difficulties in emotion regulation than HCs, which in turn predict poorer QoL.

Finally, the study of Schirda et al. (2015) examined the relationship between trait mindfulness, QoL, and difficulties in emotion regulation in a group of people with relapsing and progressive types of MS $(n=95)$. Similar to the above-mentioned studies, the authors employed the DERS (Gratz \& Roemer, 2004) for the assessment of difficulties in emotion regulation, and the WHOQoL-BREF (Skevington et al., 2004) and the Satisfaction with Life Scale (Diener et al., 1985) for the evaluation of QoL-related aspects. With regard to QoL and difficulties in emotion regulation, results of correlation analyses showed that the QoL composite score was significantly and negatively correlated with the DERS total score in people with MS. These findings suggest that MS patients with difficulties in the regulation of emotions experience less QoL.

\section{Discussion}

Studies assessing the association between socioemotional alterations and both QoL and social functioning in people with MS are still limited. Indeed, the study selection process only resulted in 8 studies that fulfilled the inclusion criteria and were included in the present review.
The majority of those studies seem to show a significant association between altered socioemotional abilities and QoL/social functioning (Isernia et al., 2019; Phillips et al., 2009, 2011, 2014; Schirda et al., 2015; van der Hiele et al., 2020). Alterations in those domains may have detrimental effects on the individuals' well-being, with negative consequences on QoL and social functioning (particularly, psychological and social dimensions of QoL and work-related aspects). Although the available evidence in MS is still limited, previous studies carried out in other clinical populations seem to support this pattern of results, showing that both social cognition deficits and difficulties with emotion regulation contribute to impaired $\mathrm{QoL}$ and social functioning in patients with different medical conditions (e.g., Cooper et al., 2015; Couture et al., 2006; Yogarajah \& Mula, 2019).

Despite this positive evidence, not all the results are consistent and other studies found no significant relationships especially between social cognition and QoL dimensions (Ciampi et al., 2018; Grothe et al., 2020).

A possible explanation for those contrasting findings may rely on the heterogeneous instruments that have been employed by the different studies. In fact, whereas for the assessment of emotion regulation abilities and QoL most authors have administered similar self-report questionnaires (e.g., the DERS and the WHOQoL-BREF, respectively), for the evaluation of social cognition skills, various measures have been adopted and this may have led to inconsistent findings. The use of different tasks (e.g., task type and complexity) can also make it difficult to compare results across the studies. In order to overcome this issue, standardized and ecological tasks, which are able to reflect how individuals behave in an enriched social context, should be employed. In this view, dynamic assessment tools, such as videotaped tasks, have been proved to have better accuracy than traditional static tests in assessing social cognition components (Dziobek et al., 2006). For instance, the only study among the ones we included in the present scoping review (Phillips et al., 2011), which used a dynamic emotion perception video task for the assessment of facial emotion recognition, was able to detect a significant association between this measure and psychological, social, and environmental domains of QoL. However, future studies employing instruments, such as the Amsterdam Dynamic Facial Expression Set (Van Der Schalk et al., 2011), the Montréal Pain and Affective Face Clips (Simon et al., 2008) and the Awareness of Social Inference Test (McDonald et al., 2006) for the assessment of social cognition components, are necessary to prove the validity of videotaped tasks to predict QoL and social functioning in people with MS more accurately.

Furthermore, although the evaluation of emotion regulation abilities has been based on the use of similar measures, these instruments are exclusively self-report. The limitations of self-report instruments are well known and 
may lead to underestimation of patients' difficulties due to self-reflection impairments typically observed in many neurological disorders. Indeed, explicit self-report measures require the respondents to be aware of their reduced ability to identify and describe feelings (e.g., Parling et al., 2010). Performance-based instruments or structured interviews, less dependent on the patient's awareness, should be employed in addition to traditional self-report measures.

Another possible explanation for the mixed findings the present review showed could be ascribed to the heterogeneous cohorts of people with MS that the different studies recruited. Indeed, patients with different disease subtypes, distinct levels of physical disability, and various stages of illness may be more or less prone to social cognitive deficits, which could have, in turn, a different impact on QoL and social functioning dimensions.

Finally, it is still a matter of debate if social cognition impairments can be considered a result of the cognitive deficits frequently observed in people with MS or if they may represent a primary sign of the disease. Nevertheless, it is clear that the combination of multiple cognitive, social, and affective symptoms may have a leverage effect on the individual's psychological well-being and day-today functioning. Indeed, the available evidence seems to show that worse QoL is negatively correlated with greater physical, cognitive and emotional disabilities, and with longer disease duration and progressive clinical course (e.g., Labiano-Fontcuberta et al., 2015; Laing et al., 2015).

The results of the present scoping should also be interpreted with caution due to some limitations of the studies we included. Firstly, most of them adopted a crosssectional design, which does not allow establishing any causal direction between socioemotional alterations and reduced QoL/social functioning. Secondly, some of those studies did not recruit also a group of HCs to be used as a comparison for MS patients.

Despite those limitations, the present scoping review represents, to the best of our knowledge, the first contribution summarizing the available evidence on the complex relation between altered socioemotional skills and reduced QoL/social functioning in patients with MS, suggesting the presence of significant associations between those constructs in MS population.

Further evidence is, however, necessary to replicate and confirm the available results. A deeper understanding of the alterations in social cognitive and emotional skills may allow researchers and clinicians to have a more comprehensive framework of the psychological symptoms associated with MS. In this way, it would be possible to plan a better medical management with tailored treatments specific for each individual's needs.
Authors' contributions MDT, MC, and LC conceived and designed the review. MDT and LC carried out the literature search and screening. MDT, MC, and LC wrote the manuscript. All authors discussed and approved the final version of the manuscript.

Funding Open access funding provided by Università degli Studi di Torino within the CRUI-CARE Agreement.

Data availability Data sharing not applicable to this article as no datasets were generated or analyzed during the current study.

Code availability Not applicable.

\section{Declarations}

Ethical approval This article does not contain any studies with human participants performed by any of the authors.

Consent to participate Not applicable.

Consent for publication Not applicable.

Conflicts of interest The authors have no relevant financial or nonfinancial interests to disclose.

Open Access This article is licensed under a Creative Commons Attribution 4.0 International License, which permits use, sharing, adaptation, distribution and reproduction in any medium or format, as long as you give appropriate credit to the original author(s) and the source, provide a link to the Creative Commons licence, and indicate if changes were made. The images or other third party material in this article are included in the article's Creative Commons licence, unless indicated otherwise in a credit line to the material. If material is not included in the article's Creative Commons licence and your intended use is not permitted by statutory regulation or exceeds the permitted use, you will need to obtain permission directly from the copyright holder. To view a copy of this licence, visit http://creativecommons.org/licenses/by/4.0/.

\section{References}

Adolphs, R. (2001). The neurobiology of social cognition. Current Opinion in Neurobiology, 11(2), 231-239. https://doi.org/10.1016/ s0959-4388(00)00202-6

Baron-Cohen, S., \& Wheelwright, S. (2004). The empathy quotient: An investigation of adults with Asperger syndrome or high functioning autism, and normal sex differences. Journal of Autism and Developmental Disorders, 34(2), 163-175. https://doi.org/ 10.1023/b:jadd.0000022607.19833.00

Baron-Cohen, S., O'Riordan, M., Jones, R., Stone, V., \& Plaisted, K. (1999). A new test of social sensitivity: Detection of faux pas in normal children and children with Asperger syndrome. Journal of Autism and Developmental Disorders, 29(5), 407-418.

Baron-Cohen, S., Wheelwright, S., Hill, J., Raste, Y., \& Plumb, I. (2001). The "Reading the Mind in the Eyes" test revised version: A study with normal adults, and adults with Asperger syndrome or high-functioning autism. Journal of Child Psychology and Psychiatry, 42(2), 241-251. https://doi.org/10.1017/S0021 963001006643

Benedict, R. H., DeLuca, J., Enzinger, C., Geurts, J. J., Krupp, L. B., \& Rao, S. M. (2017). Neuropsychology of multiple sclerosis: Looking back and moving forward. Journal of the International 
Neuropsychological Society, 23(9-10), 832-842. https://doi.org/ $10.1017 / \mathrm{s} 1355617717000959$

Benton, A. L., Hamsher, K., Varney, N. R., \& Spreen, O. (1983). Contributions to neuropsychological assessment: A clinical manual. Oxford University Press.

Bertoux, M., Delavest, M., de Souza, L. C., Funkiewiez, A., Lépine, J. P., Fossati, P., ... \& Sarazin, M. (2012). Social cognition and emotional assessment differentiates frontotemporal dementia from depression. Journal of Neurology, Neurosurgery \& Psychiatry, 83(4), 411-416. https://doi.org/10.1136/jnnp-2011-301849

Bertoux, M., Volle, E., De Souza, L. C., Funkiewiez, A., Dubois, B., \& Habert, M. O. (2014). Neural correlates of the mini-SEA (Social cognition and Emotional Assessment) in behavioral variant frontotemporal dementia. Brain Imaging and Behavior, 8(1), 1-6. https://doi.org/10.1007/s11682-013-9261-0

Bora, E., Özakbaş, S., Velakoulis, D., \& Walterfang, M. (2016). Social cognition in multiple sclerosis: A meta-analysis. Neuropsychology Review, 26(2), 160-172. https://doi.org/10.1007/ s11065-016-9320-6

Bora, E., Walterfang, M., \& Velakoulis, D. (2015). Theory of mind in behavioural-variant frontotemporal dementia and Alzheimer's disease: A meta-analysis. Journal of Neurology, Neurosurgery, and Psychiatry, 86, 714-719. https://doi.org/10.1136/ jnnp-2014-309445

Bosc, M. (2000). Assessment of social functioning in depression. Comprehensive Psychiatry, 41(1), 63-69. https://doi.org/10. 1016/s0010-440x(00)90133-0

Calabrese, P. (2006). Neuropsychology of multiple sclerosis. Journal of Neurology, 253(1), i10-i15. https://doi.org/10.1007/ s00415-006-1103-1

Chalah, M. A., \& Ayache, S. S. (2017a). Alexithymia in multiple sclerosis: A systematic review of literature. Neuropsychologia, 104, 31-47. https://doi.org/10.1016/j.neuropsychologia.2017. 07.03 .

Chalah, M. A., \& Ayache, S. S. (2017b). Deficits in social cognition: an unveiled signature of multiple sclerosis. Journal of the International Neuropsychological Society, 23(3), 266-286 https:// doi.org/10.1016/j.neuropsychologia.2017.07.034

Ciampi, E., Uribe-San-Martin, R., Vásquez, M., Ruiz-Tagle, A., Labbe, T., Cruz, J. P., ... \& Cárcamo-Rodríguez, C. (2018). Relationship between Social Cognition and traditional cognitive impairment in Progressive Multiple Sclerosis and possible implicated neuroanatomical regions. Multiple sclerosis and related disorders, 20, 122-128. https://doi.org/10.1016/j.msard.2018.01.013

Cooper, C. L., Phillips, L. H., Johnston, M., Whyte, M., \& MacLeod, M. J. (2015). The role of emotion regulation on social participation following stroke. British Journal of Clinical Psychology, 54(2), 181-199. https://doi.org/10.1111/bjc.12068

Cotter, J., Firth, J., Enzinger, C., Kontopantelis, E., Yung, A. R., Elliott, R., \& Drake, R. J. (2016). Social cognition in multiple sclerosis: A systematic review and meta-analysis. Neurology, 87(16), 1727-1736. https://doi.org/10.1212/wnl.0000000000003236

Couture, S. M., Penn, D. L., \& Roberts, D. L. (2006). The functional significance of social cognition in schizophrenia: A review. Schizophrenia Bulletin, 32(1), S44-S63. https://doi.org/10.1093/ schbul/sbl029

Diener, E., Emmons, R. A., Larsen, R. J., \& Griffin, S. (1985). The satisfaction with life scale. Journal of Personality Assessment, 49, 71-75. https://doi.org/10.1207/s15327752jpa4901_13

Di Tella, M., Castelli, L., Colonna, F., Fusaro, E., Torta, R., Ardito, R. B., \& Adenzato, M. (2015). Theory of mind and emotional functioning in fibromyalgia syndrome: an investigation of the relationship between social cognition and executive function. PloS One, 10(1), e0116542

Di Tella, M., Adenzato, M., Catmur, C., Miti, F., Castelli, L., \& Ardito, R. B. (2020). The role of alexithymia in social cognition:
Evidence from a nonclinical population. Journal of Affective Disorders, 273, 482-492

Dziobek, I., Fleck, S., Kalbe, E., Rogers, K., Hassenstab, J., Brand, M., ... \& Convit, A. (2006). Introducing MASC: a movie for the assessment of social cognition. Journal of autism and developmental disorders, 36(5), 623-636. https://doi.org/10.1007/ s10803-006-0107-0

Feinstein, A. (2004). The neuropsychiatry of multiple sclerosis. The Canadian Journal of Psychiatry, 49(3), 157-163. https://doi.org/ 10.1177/070674370404900302

Files, D. K., Jausurawong, T., Katrajian, R., \& Danoff, R. (2015). Multiple sclerosis. Primary Care: Clinics in Office Practice, 42(2), 159-175. https://doi.org/10.1016/j.pop.2015.01.007

Gratz, K. L., \& Roemer, L. (2004). Multidimensional assessment of emotion regulation and dysregulation: Development, factor structure, and initial validation of the Difficulties in Emotion Regulation Scale. Journal of Psychopathology and Behavioral Assessment, 26, 41-54. https://doi.org/10.1023/B:JOBA.0000007455. 08539.94

Green, M. F., Horan, W. P., \& Lee, J. (2015). Social cognition in schizophrenia. Nature Reviews Neuroscience, 16(10), 620-631. https:// doi.org/10.1038/nrn4005

Gross, J. J., \& John, O. P. (2003). Individual differences in two emotion regulation processes: Implications for affect, relationships and well-being. Journal of Personality and Social Psychology, 85, 248-362. https://doi.org/10.1037/0022-3514.85.2.348

Grothe, M., Opolka, M., Berneiser, J., \& Dressel, A. (2020). Testing social cognition in multiple sclerosis: Difference between emotion recognition and theory of mind and its influence on quality of life. Brain and behavior, e01925. https://doi.org/10.1002/brb3.1925

Happé, F. G. (1994). An advanced test of theory of mind: Understanding of story characters' thoughts and feelings by able autistic, mentally handicapped, and normal children and adults. Journal of Autism and Developmental Disorders, 24(2), 129-154. https:// doi.org/10.1007/BF02172093

Hobart, J., Lamping, D., Fitzpatrick, R., Riazi, A., \& Thompson, A. (2001). The multiple sclerosis impact scale (MSIS-29) a new patient-based outcome measure. Brain, 124(5), 962-973. https:// doi.org/10.1093/brain/124.5.962

Isernia, S., Baglio, F., d'Arma, A., Groppo, E., Marchetti, A., \& Massaro, D. (2019). Social mind and long-lasting disease: Focus on affective and cognitive theory of mind in multiple sclerosis. Frontiers in Psychology, 10, 218. https://doi.org/10.3389/fpsyg.2019. 00218

Krause, I., Kern, S., Horntrich, A., \& Ziemssen, T. (2013). Employment status in multiple sclerosis: Impact of disease-specific and non-disease-specific factors. Multiple Sclerosis, 19, 1792-1799. https://doi.org/10.1177/1352458513485655

Labbe, T., Ciampi, E., \& Carcamo Rodriguez, C. (2018). Social cognition: Concepts, neural basis and its role in multiple sclerosis. Neurology and Clinical Neuroscience, 6(1), 3-8. https://doi.org/ $10.1111 / \mathrm{ncn} 3.12164$

Labiano-Fontcuberta, A., Mitchell, A. J., Moreno-García, S., PuertasMartín, V., \& Benito-León, J. (2015). Impact of anger on the health-related quality of life of multiple sclerosis patients. $M u l-$ tiple Sclerosis Journal, 21(5), 630-641. https://doi.org/10.1177/ 1352458514549399

Laing, C. M., Phillips, L. H., Cooper, C. L., Hosie, J. A., \& Summers, F. (2015). Anger, quality of life and mood in multiple sclerosis. Journal of Multiple Sclerosis, 2, 1-6. https://doi.org/10.4172/ 2376-0389-1000127

Le Berre, A. P. (2019). Emotional processing and social cognition in alcohol use disorder. Neuropsychology, 33(6), 808. https://doi. org/10.1037/neu0000572 
Leslie, A. M. (1987). Pretense and representation: The origins of "theory of mind." Psychological Review, 94(4), 412. https://doi.org/ 10.1037/0033-2909.127.5.629

Lieberman, M. D. (2007). Social cognitive neuroscience: A review of core processes. Annual Review of Psychology, 58, 259-289. https://doi.org/10.1146/annurev.psych.58.110405.085654

Lischke, A., Berger, C., Prehn, K., Heinrichs, M., Herpertz, S. C., \& Domes, G. (2012). Intranasal oxytocin enhances emotion recognition from dynamic facial expressions and leaves eye-gaze unaffected. Psychoneuroendocrinology, 37(4), 475-481. https://doi. org/10.1016/j.psyneuen.2011.07.015

Luminet, O. (2009). Commentary on the paper" Is alexithymia a risk factor for major depression, personality disorder, or alcohol use disorders? A prospective population-based study". Journal of Psychosomatic Research, 68(3), 275-277. https://doi.org/10.1016/j. jpsychores.2009.07.016

McDonald, S., Bornhofen, C., Shum, D., Long, E., Saunders, C., \& Neulinger, K. (2006). Reliability and validity of The Awareness of Social Inference Test (TASIT): A clinical test of social perception. Disability and Rehabilitation, 28(24), 1529-1542. https://doi.org/ 10.1080/09638280600646185

Messinis, L., Papathanasopoulos, P., Kosmidis, M. H., Nasios, G., \& Kambanaros, M. (2018). Neuropsychological features of multiple sclerosis: impact and rehabilitation. Behavioural neurology, 2018. https://doi.org/10.1155/2018/4831647

Paparrigopoulos, T., Ferentinos, P., Kouzoupis, A., Koutsis, G., \& Papadimitriou, G. N. (2010). The neuropsychiatry of multiple sclerosis: Focus on disorders of mood, affect and behaviour. International Review of Psychiatry, 22(1), 14-21. https://doi.org/10. 3109/09540261003589323

Parling, T., Mortazavi, M., \& Ghaderi, A. (2010). Alexithymia and emotional awareness in anorexia nervosa: Time for a shift in the measurement of the concept? Eating Behaviors, 11(4), 205-210. https://doi.org/10.1016/j.eatbeh.2010.04.001

Phillips, L. H., Henry, J. D., Nouzova, E., Cooper, C., Radlak, B., \& Summers, F. (2014). Difficulties with emotion regulation in multiple sclerosis: Links to executive function, mood, and quality of life. Journal of Clinical and Experimental Neuropsychology, 36(8), 831-842. https://doi.org/10.1080/13803395.2014.946891

Phillips, L. H., Henry, J. D., Scott, C., Summers, F., Whyte, M., \& Cook, M. (2011). Specific impairments of emotion perception in multiple sclerosis. Neuropsychology, 25(1), 131. https://doi.org/ 10.1037/a0020752

Phillips, L. H., Saldias, A., McCarrey, A., Henry, J. D., Scott, C., Summers, F., \& Whyte, M. (2009). Attentional lapses, emotional regulation and quality of life in multiple sclerosis. British Journal of Clinical Psychology, 48(1), 101-106. https://doi.org/10.1348/ $014466508 \times 379566$

Planche, V., Gibelin, M., Cregut, D., Pereira, B., \& Clavelou, P. (2015). Cognitive impairment in a population-based study of patients with multiple sclerosis: Differences between late relapsing-remitting, secondary progressive and primary progressive multiple sclerosis. European Journal of Neurology. https://doi.org/10.1111/ene. 12715

Prakash, R. S., Snook, E. M., Lewis, J. M., Motl, R. W., \& Kramer, A. F. (2008). Cognitive impairments in relapsing-remitting multiple sclerosis: A meta-analysis. Multiple Sclerosis, 14, 1250-1261. https://doi.org/10.1177/1352458508095004

Premack, D., \& Woodruff, G. (1978). Does the chimpanzee have a theory of mind? Behavioral and Brain Sciences, 1(4), 515-526. https://doi.org/10.1017/S0140525X00076512

Richards, A., French, C. C., Calder, A. J., Webb, B., Fox, R., \& Young, A. W. (2002). Anxiety-related bias in the classification of emotionally ambiguous facial expressions. Emotion, 2(3), 273. https:// doi.org/10.1037/1528-3542.2.3.273
Schirda, B., Nicholas, J. A., \& Prakash, R. S. (2015). Examining trait mindfulness, emotion dysregulation, and quality of life in multiple sclerosis. Health Psychology, 34(11), 1107. https://doi.org/ 10.1037/hea0000215

Schwartz, C., \& Frohner, R. (2005). Contribution of demographic, medical, and social support variables in predicting the mental health dimension of quality of life among people with multiple sclerosis. Health \& Social Work, 30(3), 203-212. https://doi.org/ 10.1093/hsw/30.3.203

Senju, A. (2013). Atypical development of spontaneous social cognition in autism spectrum disorders. Brain and Development, 35(2), 96-101. https://doi.org/10.1016/j.braindev.2012.08.002

Siegert, R. J., \& Abernethy, D. A. (2005). Depression in multiple sclerosis: A review. Journal of Neurology, Neurosurgery \& Psychiatry, 76(4), 469-475. https://doi.org/10.1136/jnnp.2004.054635

Simeoni, M. C., Auquier, P., Fernandez, O., Flachenecker, P., Stecchi, S., Constantinescu, C. S., ... \& Pelletier, J. (2008). Validation of the multiple sclerosis international quality of life questionnaire. Multiple Sclerosis Journal, 14(2), 219-230. https://doi.org/10. 1177/1352458507080733

Simon, D., Craig, K. D., Gosselin, F., Belin, P., \& Rainville, P. (2008). Recognition and discrimination of prototypical dynamic expressions of pain and emotions. PAIN®, 135(1-2), 55-64. https://doi. org/10.1016/j.pain.2007.05.008

Skevington, S. M., Lotfy, M., \& O'Connell, K. A. (2004). The World Health Organisation's WHOQOL-BREF quality of life assessment: Psychometric properties and results of the international field trial. A report from the WHOQOL group. Quality of Life Research, 13, 299-310. https://doi.org/10.1023/b:qure.00000 18486.91360 .00

Slessor, G., Phillips, L. H., \& Bull, R. (2007). Exploring the specificity of age differences in Theory of Mind tasks. Psychology and Aging, 22, 639-643. https://doi.org/10.1037/0882-7974.22.3.639

Taylor G. J., Bagby R. M., \& Parker J. D. A. (1997). Disorders of Affect Regulation: Alexithymia in Medical and Psychiatric Illness. Cambridge University Press.

Tricco, A. C., Lillie, E., Zarin, W., O'Brien, K. K., Colquhoun, H., Levac, D., ... \& Straus, S. E. (2018). PRISMA extension for scoping reviews (PRISMA-ScR): checklist and explanation. Annals of internal medicine, 169(7), 467-473. https://doi.org/10.7326/ $\mathrm{m} 18-0850$

van der Hiele, K., van Egmond, E. E. A., Jongen, P. J., van der Klink, J. J. L., Beenakker, E. A. C., van Eijk, J. J. J., ... \& van Gorp, D. A. M. (2020). Empathy in multiple sclerosis-correlates with cognitive, psychological and occupational functioning.Multiple Sclerosis and Related Disorders. https://doi.org/10.1016/j.msard. 2020.102036

Van Der Schalk, J., Hawk, S. T., Fischer, A. H., \& Doosje, B. (2011). Moving faces, looking places: validation of the Amsterdam Dynamic Facial Expression Set (ADFES). Emotion, 11(4), 907. https://psycnet.apa.org/doi/10.1037/a0023853

Vickrey, B., Hays, R. D., Harooni, R., Myers, L. W., \& Ellison, G. W. (1995). A health-related quality of life measure for multiple sclerosis. Quality of Life Research, 4(3), 187-206. https://doi.org/ $10.1007 / \mathrm{bf02260859}$

Washburn, D., Wilson, G., Roes, M., Rnic, K., \& Harkness, K. L. (2016). Theory of mind in social anxiety disorder, depression, and comorbid conditions. Journal of Anxiety Disorders, 37, 71-77. https://doi.org/10.1016/j.janxdis.2015.11.004

Yogarajah, M., \& Mula, M. (2019). Social cognition, psychiatric comorbidities, and quality of life in adults with epilepsy. Epilepsy \& Behavior, 100, 106321. https://doi.org/10.1016/j.yebeh. 2019.05.017

Young, A., Perrett, D., Calder, A., Sprengelmeyer, R., \& Ekman, P. (2002). Facial Expressions of Emotion - Stimuli and Tests (Software Manual v2.1 Ed.). Thames Valley Test Company. 
Publisher's note Springer Nature remains neutral with regard to jurisdictional claims in published maps and institutional affiliations. 